\title{
Nose and Sinus Air Flow Model
}

\author{
R. De Luca1, M. Gamerra², G. Sorrentino², E. Cantone ${ }^{3,4}$ \\ ${ }^{1}$ Department of Physics "E. R. Caianiello", University of Salerno, Fisciano, Italy \\ ${ }^{2}$ Divisione di Otorinolaringoiatria, Ospedale "S. Leonardo"-A.S.L. NA 3 sud, Castellammare di Stabia, Italy \\ ${ }^{3}$ Department of Neuroscience, Reproductive and Odontostomatologic Science, ENT Unit, "Federico II" \\ University, Naples, Italy \\ ${ }^{4}$ Department of Molecular Medicine and Medical Biotechnology, “Federico II" University, Naples, Italy \\ Email: rdeluca@unisa.it
}

Received 10 April 2014; revised 10 May 2014; accepted 17 May 2014

Copyright (C) 2014 by authors and Scientific Research Publishing Inc.

This work is licensed under the Creative Commons Attribution International License (CC BY).

http://creativecommons.org/licenses/by/4.0/

(c) (i) Open Access

\begin{abstract}
Air flow in nose and sinuses is studied by means of a simple model based on the steady-state ideal fluid flow assumption and repeated use of Bernoulli's equation. In particular, by describing flow of air drawn in through the vestibulumnasi during inspiration, we investigate how ventilation of the maxillary sinus is affected by surgical removal of part of the lateral walls of the nasal cavity close to the ostiummeatal complex. We find that, according to the model proposed, removal of tissues from this inner part of the nasal cavity may cause a decrease of the flux rate from the maxillary sinus.
\end{abstract}

\section{Keywords}

Air Flow Model, Nose and Sinus, Bernoulli's Equation

\section{Introduction}

The human ventilation system works by means of gaseous exchanges, which takes place between the nose and sinus cavities, and between the latter and the blood circle through the mucosa [1]. During respiratory acts, air flowing in the nasal cavity reaches the paranasal sinuses through the hosts and their ducts [2]. Therefore, a correct anatomical and physiological equilibrium which is able to generate effective pressure gradients inside the nasal cavity plays an important role in the ventilation of the sinus cavities. During a single inhalation, air flows from the vestibulum towards the coana and produces, by "sucking effect", negative pressures into the ostiummetal complex [3]. As a result, at the beginning of each nasal inhalation action, a negative nose pressure is generated, in such a way that air flows out from the sinus cavities, due to the effect of aspiration. Air successively re-enters these cavities when the inspiration phase ends, and thus one of the continuous life-long respiratory cycles is completed. A fundamental role for a correct ventilation is thus played by the anatomical conformation of 
the ostiummeatal complex [4].

In sinus physiology, air exchange is also regulated by diffusive molecular mechanisms related to the chemical and physical characteristics of the inhaled air mixture. The correct and continuous sinus ventilation due to the physical phenomenon described above is the reason why air in the sinus cavities is always in motion. The shape of the nasal cavity can be assimilated to that of a tube to which, under stationary conditions and ideal fluid flow, Bernoulli's equation can be applied. Under the hypothesis of applicability of Bernoulli's equation, therefore, the single particles of the fluid are taken to describe laminar trajectories with no energy loss in all ducts.

In the present work, the problem of air flow through the ostiummeatal complex coming from the maxillary sinus is studied by means of repeated use of Bernoulli's equation. The work is thus organized as follows. In the following section, we give a detailed description of the model adopted. In the third section, we solve the model equations by means of a first-order perturbation approach, deriving a direct analytic dependence between the flux rate in the infundibulum and the nose's effective section. Conclusions are drawn in the last.

\section{The Model}

We consider the schematic model of the ostium-meatal complex reported in Figure 1. In this figure we schematize the maxillary sinus as a spherical cavity (M), in which air at the atmospheric pressure is present. The ostiummeatal complex is seen as a short duct linking the nasal cavity $(\mathrm{N})$ to the maxillary sinus. In the schematic representation of Figure 1, during inspiration air enters the nose through the vestibulumnasi and comes out through the coana (C-posterior). This air-flow produces a depression in $\mathrm{N}$ close to the ostiummeatal complex, sucking air from the maxillary sinus.

By denoting with $S_{0}$ and $S_{1}$ the effective sections of the inner and outer portions of the nasal cavity, respectively, and by $S_{2}$ the effective section of the ostiummeatal complex, we assume that air behaves as an ideal fluid [5] through these cavities. Air flows with velocities $V_{0}, V_{1}$, and $V_{2}$ through the correspondingly indexed sections, so that, by continuity equation we can write the following relations for the flow rates in these sections:

$$
S_{0} V_{0}=S_{1} V_{1}+S_{2} V_{2} \text {. }
$$

By extending Bernoulli's equation to a Y-shaped tube [6], in the absence of gravitational effects, we may write:

$$
\left(p_{0}+\frac{1}{2} \rho V_{0}^{2}\right) \Delta \tau_{0}=\left(p_{1}+\frac{1}{2} \rho V_{1}^{2}\right) \Delta \tau_{1}+\left(p_{2}+\frac{1}{2} \rho V_{2}^{2}\right) \Delta \tau_{2} .
$$

where $p_{0}$ and $p_{1}$ are the pressures in the inner and outer portions of the nasal cavity, respectively, and $p_{2}$ is the

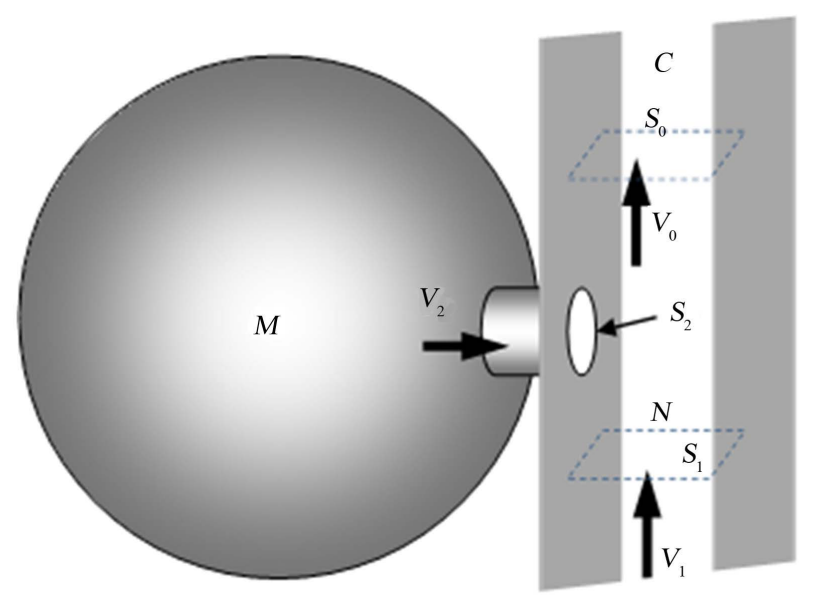

Figure 1. A schematic representation of the ostium-meatal complex. Air, drawn trough the vestibulim nasi, enters the nasal cavity $(N)$ with velocity $V_{1}$. Air fromthe ostium-meatal complex, having velocity $V_{2}$, mixes with inhaled air in the inner nasal cavity towards the coana $(C)$ during inspiration. Air from the maxillary sinus $(M)$ is assumed to be at rest at atmosperic pressure $p_{a}$. 
pressure in the ostiummeatal complex. Moreover, in Equation (2) $\rho$ is the density of air, $\Delta \tau_{1}$ is the volume of the inhaled air, $\Delta \tau_{2}$ is the volume of air drawn from the ostiummeatal complex and $\Delta \tau_{0}$ is the volume of air flowing in the coana, given by:

$$
\Delta \tau_{0}=\Delta \tau_{1}+\Delta \tau_{2}
$$

Notice that Equation (3) is a mere consequence of Equation (1). In this way, Equation (2) can be rewritten in terms of the volume flow rates, defined in Equation (1), as follows:

$$
\left(p_{0}+\frac{1}{2} \rho V_{0}^{2}\right) V_{0} S_{0}=\left(p_{1}+\frac{1}{2} \rho V_{1}^{2}\right) V_{1} S_{1}+\left(p_{2}+\frac{1}{2} \rho V_{2}^{2}\right) V_{2} S_{2}
$$

By Bernoulli's equation, considering a point in $\mathrm{M}$ and a point in the ostiummeatal complex, we can write

$$
p_{a}=p_{2}+\frac{1}{2} \rho V_{2}^{2}
$$

where $p_{a}$ is the atmospheric pressure. Moreover, by assuming that air flowing in the vestibulumnasi is drawn at constant velocity $V_{L}$ during inspiration, we have:

$$
\begin{gathered}
k_{V}=p_{a}+\frac{1}{2} \rho V_{V}^{2}=p_{1}+\frac{1}{2} \rho V_{1}^{2} \\
S_{1} V_{1}=S_{V} V_{V} .
\end{gathered}
$$

where $k_{V}$ is a constant and $S_{V}$ is the effective section of the vestibulumnasi. In this respect, we need to specify that the assumption on $V_{V}$ is correlated to the patient's needs of air intake, which can safely be assumed to be constant. On the other hand, the value of $k_{V}$ is the sum of the atmospheric pressure and of the dynamical pressure term linked to $V_{V}$. This term, though varying from individual to individual, remains constant for a single patient. By now considering Equations (5) and (6a-b), we may rewrite Equation (4) in the following way:

$$
\left(p_{0}+\frac{1}{2} \rho V_{0}^{2}\right) V_{0} S_{0}=k_{V} V_{V} S_{V}+p_{a} \Phi_{I},
$$

where $\Phi_{I}=S_{2} V_{2}$ is the flux rate inside the ostiummeatal complex. By implicitly differentiating Equation (7) and by noticing that, by Equations (1) and (6b), $d \Phi_{I}=d\left(S_{0} V_{0}\right)$, we write:

$$
\left(p_{a}-p_{0}-\frac{1}{2} \rho V_{0}^{2}\right) d \Phi_{I}=S_{0} V_{0} d p_{0}+\rho S_{0} V_{0}^{2} d V_{0}
$$

where the differential quantity $d \Phi_{I}$ accounts for an infinitesimal variation of the flux rate in the ostiummeatal complex solely due to a corresponding infinitesimal variation $S_{0}$ of the coana effective section. In order to obtain a set of equations by which we can directly relate $d \Phi_{I}$ with $d S_{0}$, we introduce one further assumption, i.e., that air can flow at the same temperature inside the nasal cavity before and after the variation $d S_{0}$ has taken place, so that:

$$
d\left(S_{0} V_{0} p_{0}\right)=0 \Rightarrow d p_{0}\left(S_{0} V_{0}\right)=-p_{0} d \Phi_{I}
$$

By now substituting the above expression in Equation (8) we have:

$$
\left(p_{a}-\frac{1}{2} \rho V_{0}^{2}\right) d \Phi_{I}=\rho S_{0} V_{0}^{2} d V_{0}
$$

We notice that in Equation (10) $d V_{0}$ can be related to $d S_{0}$ by equating the expression $d \Phi_{I}=d\left(S_{0} V_{0}\right)$, following from Equations (1) and (6b), to the expression for $d \Phi_{I}$ obtained from Equation (10). In this way, we have:

$$
\frac{d V_{0}}{V_{0}}\left(\frac{p_{a}-\frac{3}{2} \rho V_{0}^{2}}{p_{a}-\frac{1}{2} \rho V_{0}^{2}}\right)=-\frac{d S_{0}}{S_{0}} .
$$

By considering Equation (11), it is now not difficult to show that an implicit functional relation of $V_{0}$ in terms of $S_{0}$ is given by the following expression: 


$$
\left(p_{a}-\frac{1}{2} \rho V_{0}^{2}\right) V_{0}=\frac{k}{S_{0}}
$$

where $k$ is a constant parameter referring to a specific group of patients. In order to obtain a meaningful order of magnitude for $k$, we might consider the main term in Equation (12), namely, the product $p_{a} V_{0} S_{0}$. In this way, we notice that, for $V_{0} \approx 5.0 \mathrm{~cm} / \mathrm{s}$ and for $S_{0} \approx 100 \mathrm{~mm}{ }^{2}$, we have $k$ of about $5.0 \mathrm{~N} \cdot \mathrm{m} \cdot \mathrm{s}^{-1}$. Equation (12) can now be inverted either numerically, either analytically, in order to obtain $V_{0}$ vs. $S_{0}$ curves, which we show in Figure 2 for various values of the constant parameter $k$. Considering now Equations (10) and (11), we can set:

$$
d \Phi_{I}=-\frac{\rho V_{0}^{3}}{\left(p_{a}-\frac{3}{2} \rho V_{0}^{2}\right)} d S_{0},
$$

which directly relates the infinitesimal change $d \Phi_{I}$ to $d S_{0}$. By means of Equation (12) it could be possible to find how the flux rate $\Phi_{I}$ depends explicitly on $S_{0}$. However, in the following section, we shall adopt a perturbation approach to obtain this dependence.

\section{Perturbation Solution and Approximated Results}

In the previous section we have obtained Equations (12) and (13), which represent the solution to the proposed problem of finding how the flux rate $\Phi_{I}$ inside the ostiummeatal complex varies with respect to the effective area of the coana $S_{0}$. Even though an analytic expression for such dependence can in principle be found, it is not convenient to proceed in this way, since a perturbation approach can be adopted, given that the dynamic pressure $\rho V_{0}^{2} / 2$ is, for this type of system, much smaller than $p_{a}$. With this in mind, to first order in the term $\rho V_{0}^{2} / 2 p_{a}$, Equations (12) and (13) can be written in the following way:

$$
\begin{aligned}
V_{0} S_{0} p_{a} & =k\left(1+\frac{\rho V_{0}^{2}}{2 p_{a}}\right), \\
d \Phi_{I} & =-\frac{\rho V_{0}^{3}}{p_{a}} d S_{0} .
\end{aligned}
$$

Solving for $V_{0}$ in Equation (14a), we have:

$$
V_{0}=\frac{S_{0} p_{a}^{2}}{\rho k}\left(1-\sqrt{1-\frac{2 \rho k^{2}}{p_{a}^{3} S_{0}^{2}}}\right),
$$

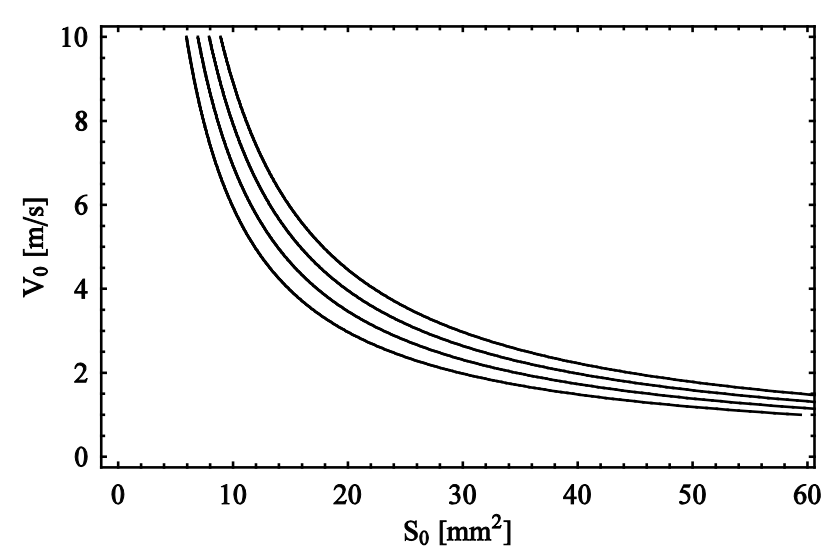

Figure 2. The velocity $V_{0}$ of air in the inner part of the nasal cavity as a function of the effective area $S_{0}$ for $\rho=1.29 \mathrm{~kg} \cdot \mathrm{m}^{-3}$, $p_{a}=1.0 \mathrm{~atm}$ and for the following values of the parameter $k$ (from bottom to top): $6,7,8,9 \mathrm{~N} \cdot \mathrm{m} \cdot \mathrm{s}^{-1}$. 
In this expression we chose the minus sign in front of the square root, since it correctly gives a decreasing behavior of $V_{0}$ for small values of $S_{0}$ and take $S_{0}{ }^{2}>2 \rho k^{2} / p_{a}{ }^{3}$. In Figure 2, we show the velocity $V_{0}$ of air in the inner part of the nasal cavity as a function of the effective area $S_{0}$, as given by Equation (15), for $\rho=1.29 \mathrm{~kg} \cdot \mathrm{m}^{-3}$, $p_{a}=1.0 \mathrm{~atm}$, and the following values of the parameter $k$ (from bottom to top): $6,7,8,9 \mathrm{~N} \cdot \mathrm{m} \cdot \mathrm{s}^{-1}$.

Substituting now Equation (15) in Equation (14), we finally have:

$$
d \Phi_{I}=-\frac{p_{a}^{5}}{\rho^{2} k^{3}}\left(S_{0}-\sqrt{S_{0}^{2}-\frac{2 \rho k^{2}}{p_{a}^{3}}}\right)^{3} d S_{0},
$$

By now calling $A=p_{a}{ }^{5} / \rho^{2} \cdot k^{3}$ and $B=2 \rho k^{2} / p_{a}{ }^{3}$, we may easily integrate Equation (16), obtaining

$$
\Phi_{I}=-A\left(S_{0}^{4}-S_{0}\left(S_{0}^{2}-B\right)^{3 / 2}-\frac{3 B S_{0}^{2}}{2}\right)+c,
$$

where $c$ is a constant. Since we are only interested in finite variations of $\Phi_{I}$, we do not need to calculate the constant $c$. Moreover, we make take Equation (16) as the first-order approximation of the flux rate variation $\Delta \Phi_{I}$ due to a finite variation of the nasal cavity effective section $\Delta S_{0}$, so that

$$
\Delta \Phi_{I} \approx-\frac{p_{a}^{5}}{\rho^{2} k^{3}}\left(S_{0}-\sqrt{S_{0}^{2}-\frac{2 \rho k^{2}}{p_{a}^{3}}}\right)^{3} \Delta S_{0}
$$

The above equation can be considered as an approximation to the solution to the problem we considered in the present work. For some typical values of the parameters in Equation (18) and for an effective section of about one tenth of a square centimeter, the coefficient linking $\Delta \Phi_{I}$ and $\Delta S_{0}$ is found to be numerically equal to $8.6 \times 10^{-3} \mathrm{~m} \cdot \mathrm{s}^{-1}$. In this way, by taking, for example, $\Delta S_{0}=1.0 \mathrm{~mm}^{2}$, we have $\Delta \Phi_{I}=-8.6 \mathrm{~mm}^{3} \cdot \mathrm{s}^{-1}$. The derivative $d \Phi_{I} / d S_{0}$, as it can be obtained from Equation (16), is represented, as a function of $S_{0}$, in Figure 3 for $\rho=$ $1.29 \mathrm{~kg} \cdot \mathrm{m}^{-3}, p_{a}=1.0 \mathrm{~atm}$, and for the following values of the parameter $k$ (from top to bottom): $6,7,8,9$ $\mathrm{N} \cdot \mathrm{m} \cdot \mathrm{s}^{-1}$. From Figure 3 and from Equation (18) it can be seen that the derivative $d \Phi_{I} / d S_{0}$ is negative, so that any positive variation of $S_{0}$ causes a decrease of the flux rate in the ostiummeatal complex. In this respect, one might argue that surgical removal of anatomical structures close to the ostiummeatal complex might worsen the ventilation functional efficacy of the maxillary sinus. This particular aspect has been already observed when analyzing the effectiveness of sinus ventilation with the aid of nose and sinus manometric measurements [7]. In these particular studies it was found that better functional results can be achieved by using a conservative surgical technique preserving nose anatomy rather than a non-conservative endoscopic surgery.

\section{Conclusion}

Air flow in the nasal cavity is studied by means of a simple model resting on the stationary ideal fluid flow hy-

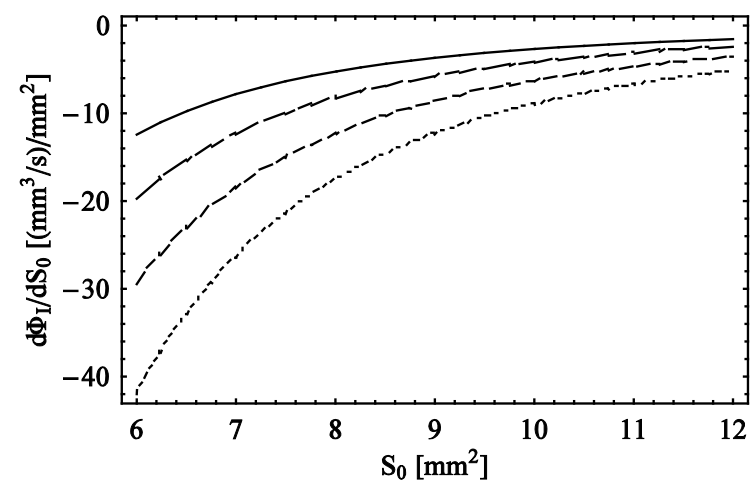

Figure 3. The derivative $d \Phi_{I} / d S_{0}$ represented as a fiunction of $S_{0}$ for $\rho=1.29 \mathrm{~kg} \cdot \mathrm{m}^{-3}, p_{a}=1.0 \mathrm{~atm}$ and for the following values of the parameter $k$ (from top to bottom): $6,7,8$, $9 \mathrm{~N} \cdot \mathrm{m} \cdot \mathrm{s}^{-1}$. The units of the derivative are purposely expressed as " $\left(\mathrm{mm}^{3} / \mathrm{s}\right) / \mathrm{mm}^{2}$ " instead of " $\mathrm{mm} / \mathrm{s}$ ". 
pothesis. Under these assumptions, Bernoulli's equation can be used. The model might thus be used to give an elementary description of nose and sinus ventilation. Under these simplifying assumptions, the present analysis predicts that the flow rate of air sucked from the maxillary sinus towards the nasal cavity decreases as the area close to the ostiummeatal complex increases. In this respect, one might argue that surgical removal of anatomical structures close to the ostiummeatal complex may worsen the ventilation functional efficacy of maxillary sinus, in accordance with experimental observations.

\section{References}

[1] Stammberger, H. and Hawke, M. (1993) Essential of Functional Endoscopic Sinus Surgery. Mosby, St. Louis.

[2] Messerklinger, W. (1978) Endoscopy of the Nose. Urban \& Schwarzenberg, Baltimore.

[3] Passali, D. (2003) Le rinosinusiti. Pacini Ed., Pisa.

[4] Bruno, R., Gamerra, M., Porpora, D., Pagano, G., Napolitano, B. and Bruno, E. (2004) Nose: Aesthetics and Function. Anales Otorrinolaringológicos Ibero-Americanos, 31, 307-323.

[5] Halliday, D., Resnick, R. and Walker, J. (2001) Foundamentals of Physics. 5th Edition, John Wiley \& Sons, New York.

[6] Gamerra, M. and De Luca, R. (2004) Un modello semplice per descrivere gli effetti sulla ventilazione sinusale del flusso d'aria immessa nella cavità nasale. Giornale di Fisica, XLV, 225-228.

[7] Gamerra, M., De Luca, R., Pagano, G., Merone, M. and Cassano, M. (2013) The Nose and Sinus Manometry: A Biophysical Model Applied to Functional Endoscopic Sinus Surgery. Journal of Biological Regulators and Homeostatic Agents, 27, 1021-1027. 
Scientific Research Publishing (SCIRP) is one of the largest Open Access journal publishers. It is currently publishing more than 200 open access, online, peer-reviewed journals covering a wide range of academic disciplines. SCIRP serves the worldwide academic communities and contributes to the progress and application of science with its publication.

Other selected journals from SCIRP are listed as below. Submit your manuscript to us via either submit@scirp.org or Online Submission Portal.
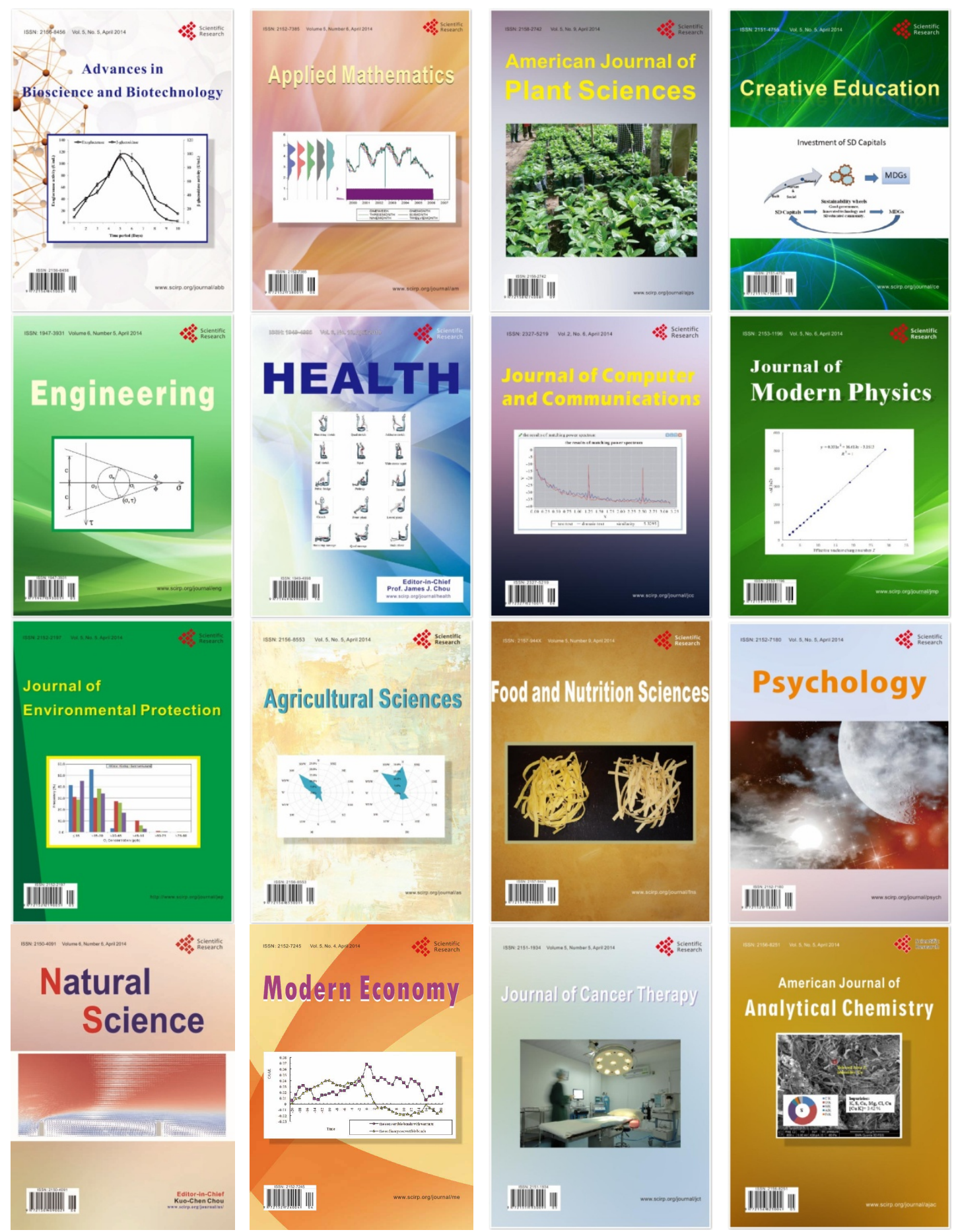